\title{
Friedensmodelle in Asien
}

\author{
Miao-ling Hasenkamp*
}

\begin{abstract}
As the gravity of world politics moves toward Asia following the rise of China and India as influential players, the Asian actors are asked to assert their role in promoting regional and world peace. What kind of Asian-style peace models can be derived from their post-war history? The essay discusses the idea of "peace in different Asian cultural backgrounds and examines the relevance and problems of five important peace models (the Confucian model with its authoritarian culture, the Japanese model with its modernization, the Indian model in showing democracy and unity in diversity, the Indonesian Muslim democracy model and the economic integration model). The essay concludes that despite the merits of these models, they suffer from serious deficits. Asia lacks a critical and comprehensive review of its own past not only in the bilateral relations among states (the question of Japan's war responsibility toward its neighbors during World War II), but also in domestic affairs (the government-made atrocities in China and Indonesia).
\end{abstract}

Keywords: Konfuzianismus, Einheit in Vielfalt, Indonesischer Islam, wirtschaftliche Integration, Aufarbeitung der Vergangenheit

\section{Einleitung}

$\mathrm{W}$

enn sich die Gewichte in Demographie, internationaler Politik und Weltwirtschaft dem jetzigen Trend folgend weiter gen Asien verschieben, so ist auf Dauer ein größerer asiatischer Beitrag zum Frieden unentbehrlich. Und wegen der wachsenden Bedeutung ihrer Region werden asiatische Akteure auch die Inhalte dieses »Friedens « weit stärker selbst prägen.

Was verstehen Chinesen, Japaner, Koreaner, Inder, Indonesier etc. unter »Frieden «? Welche Friedenserfahrungen haben die asiatischen Staaten gemacht? Gibt es hier Barrieren, welche ein Friedenprojekt oder das Bemühen um langfristigen Frieden auf regionaler Ebene beeinträchtigen oder gar verhindern können? Der Aufsatz erklärt zuerst den Begriff »Frieden « in verschiedenen asiatischen Kulturkreisen, stellt anschließend die wichtigsten Modelle dar (das konfuzianische Modell, das japanische Modell, das Indien-Modell, das indonesische Demokratie-Modell und das wirtschaftliche Integrationsmodell) und diskutiert ihre Relevanz und Problematik.

\section{Frieden als Begriff in verschiedenen asia- tischen Kulturkreisen}

Für viele Asiaten gilt Frieden als eine kosmische und philosophische Zielvorstellung mit hohem moralischem Anspruch, in der jeder Mensch durch Prozesse der Reflexion und Leidens-Transformation die Harmonie mit dem Kosmos, mit der Natur und vor allem mit den Mitmenschen anstreben soll und kann. ${ }^{2}$ Im indischen Sanskrit heißt shanti Frieden und bedeu-

* Dr. Miao-ling Hasenkamp ist Lehrbeauftragte des Instituts für Politikwissenschaft (IfPol) an der Westfälischen Wilhelms-Universität Münster.

2 Vgl. Baidyanath Saraswati, »Introduction. Diffusing Glory with Peace, « 1999, Indira Gandhi National Centre for the Arts, New Delhi, <http://ignca.nic. in/cd_09003.htm>. tet einen sublimen ontologischen Zustand inniger Ruhe, in dem Körper und Seele durch die moralische und reflexive Vernunft in Einklang miteinander sind. ${ }^{3}$ Ähnlich beinhaltet Friede im chinesischen I-Ging das »Buch der Wandlungen « die Vereinigung der Kräfte zwischen dem Himmel (Yan) und der Erde (Yin) in inniger Harmonie. Die Ordnung des Himmels, die ewig gleichbleibende, gibt auch dem Handeln auf Erden seine Maße vor. ${ }^{4}$ Es ist das Tao, das Gesetz des Seins und der Wirklichkeit, das die Menschen erkennen und im Handeln aufnehmen müssen. Das Tao ist ebenso kosmisches wie sittliches Gesetz. Es verbürgt nicht nur die Harmonie von Himmel und Erde, sondern auch die Harmonie des politischen und gesellschaftlichen Lebens. ${ }^{5}$

Allerdings verblasst eine solche Zielvorstellung gegenüber der Realität. Chinesische Dichter schilderten das ewige Dilemma zwischen Krieg und Frieden und äußerten ihren Abscheu gegenüber kriegerischer Politik. ${ }^{6}$ Die japanische Kultur bezieht den Begriff des Leidens ein und betrachtet ihn als Heilungsbzw. Transformationsprozess im Sinne des endgültigen Guten. Das Leiden wie Krankheit und Opfer der Gewalt verwandelt sich durch Mitleiden und Besinnung und schenkt dadurch den Betroffenen und der Gesellschaft heilende Kräfte. Für M. Kasai kann die Perspektive und Erfahrung von der Kultur des Friedens nicht vollständig sein ohne den Bezug auf solche Leidens- und Heilungserfahrungen. ${ }^{7}$

Aus kultur-geographischer Sicht ist Asien vor allem der Ort, wo die großen Weltreligionen entstanden, Blütezeiten und Rückschläge erlebten. Wie B. Saraswati andeutet, war Indien

3 Über die Zivilisation des Hinduismus siehe Jnanendranath Ray, Eternal Relevance of Sanatana Dharma: Hinduism and Neo-Hinduism to Mankind, Kolkata, Firma KLM, 2004, xiv, 510 p.

4 Siehe Richard Wilhelm, I-Ging. Das Buch der Wandlungen, München: Diederichs, 1998

5 Laotse, Tao Te King und Tschuang Tse, Dichtung und Weisheit, gebundene Ausgabe, Marixverlag, 2004.

6 Saraswati, Diffusing Glory with Peace, 1999.

7 Zitiert in Saraswati, ebd. 
das Land, wo Buddha erklärte, sich von Angst und Leiden befreit zu haben. ${ }^{8}$ Der Buddhismus sieht die ganze Welt als einen Kreislauf des Leidens; das Heil ist nicht im Kosmos, sondern im Heraustreten aus ihm zu suchen. Insofern das Heilsstreben nicht-weltlich - aufs Nirwana gerichtet - gefasst ist, hat der Buddhismus keine weltliche politische Vision geschaffen. Dies ist vermutlich der Hauptgrund dafür, dass es in der Geschichte des Buddhismus auch keinen Fall eines buddhistisch begründeten Angriffskriegs gab. ${ }^{9}$ Hinzu kommt die Tatsache, dass der Buddhismus sich, im Unterschied zu den monotheistischen Religionen, in keinem Land Asiens als einzige Religion durchgesetzt hat, sondern die bestehenden oder sogar neu hinzukommenden Religionen (Islam, Christentum) tolerierte oder gar in sich aufnahm.

Das Christentum hat viele Teile Asiens kulturell und politisch beeinflusst. Die biblische Tradition enthält auch diesen Grundsatz: Innerer und äußerer Friede sind nur möglich, wenn die wesentlichen Rechtsgüter des Menschen und der Gemeinschaft gesichert sind. ${ }^{10}$ Nach dem Neuen Testament ist der Christ an die Rechtsordnung des Staates als eine sittliche Ordnung gebunden, sofern die Staatsorgane Frieden und Recht garantieren. Zugleich ist eine Begrenzung des Staates enthalten: Er hat seinen Bereich, den er nicht überschreiten darf. Dieses Prinzip geht zurück auf eine Synthese von kosmischer und geschichtlicher Sicht im Friedensverständnis des Christentums. Die christliche Lehre sieht die Geschichte nicht einfach kosmisch, sondern interpretiert sie als Dynamik von Gut und Böse in fortschreitender Bewegung, so J. Ratzinger. ${ }^{11}$ Die Dynamisierung der Geschichte verdeutlicht die sittlichen Maßstäbe der Politik und zeigt die Grenzen der politischen Macht an. Hierfür ist das Leiden Christi von zentraler Bedeutung. Der gekreuzigte Christus zeigt die Grenze staatlicher Gewalt an und zeigt, wo seine Rechte enden und der Widerstand im Leiden zur Notwendigkeit wird.

Der Islam als gestaltende Kraft ist auch in Asien wichtig, bedenkt man, dass die bevölkerungsstärksten islamischen Länder wie Indonesien, Bangladesch und Pakistan in Südost- bzw. Südasien liegen. ${ }^{12}$ Auf der Grundlage der Moral des Korans war der Islam von Anfang an eine bewegende Kraft für eine soziale Reform mit dem Ziel, eine ethisch gerechte Gesellschaft zu schaffen. Für Muslime gehören Glaube, Wissen, gute Tat und Gerechtigkeit zusammen. ${ }^{13}$ Verantwortung für sich und andere wird betont. Im weiteren Sinne bildet die Treue gegenüber Allahs Geboten die Grundlage der islamischen Sicht von Re-

8 Ebd.

9 Allerdings gibt es aus dem vergangenen Jahrhundert Beispiele, wo kriegerische Handlungen als Akte der Verteidigung der buddhistischen Kultur oder der Kultur Asiens gerechtfertigt wurden. Vgl. Franz-Johannes Litsch, »Der Beitrag des Buddhismus zur Uberwindung der Gewalt, « Vortrag auf dem Seminar der Landeszentrale für Politische Bildung: »Brücken in die Zukunft Über den Zusammenhang von Religions- und Weltfrieden«, 20.4. 2002, Magdeburg.

10 Siehe Joseph Kardinal Ratzinger, Werte in Zeiten des Umbruchs. Die Herausforderungen der Zukunft bestehen, Freiburg/Basel/Wien: Herder, 2005, S. 20 .

11 Die Dynamisierung der Geschichte schildert besonders das Buch Daniel.

12 Klaus H. Schreiner (Hg.), Islam in Asien, Horlemann Verlag, 2001.

13 Über die Grundlage des Islam, das Konzept »Frieden« im Islam siehe »The concept of freedom in Islam «, presented by the Al-Balagh Foundation, unter: <http://home.swipnet.se/islam/articles/concept-freedom.htm>; Harun Yahya, »Der Islam ist nicht die Quelle des Terrorismus, sondern seine Lösung«, unter: <http://www.harunyahya.com/de/artikel08_mainartikel.php> (12.8.2005). gierung, nämlich, die Errichtung einer toleranten, gerechten und menschlichen Herrschaft. Für H. Yahya führt die Kombination der moralischen und sozialen Philosophie dazu, dass es in der Geschichte des Islams eine große Anzahl von gerechten, barmherzigen, demütigen und reifen Herrschern gab. ${ }^{14}$

Asien weist konkurrierende geistliche Traditionen, Weltanschauungen und Glaubensbekenntnisse auf, für die sich der Positivgehalt von Frieden nur im Rückgriff auf ein je bestimmtes Politik- und Gesellschaftsverständnis festlegen lässt. Frieden wird in diesen Traditionen als kosmisches bzw. göttliches Ordnungsprinzip, als überhistorischer Ausdruck eines leidensfreien (Ideal-) Zustands begriffen. Je nach den moralisch-ethischen Grundannahmen und Normen des Individuums sowie gesellschaftlichen Wertvorstellungen wird der Friedensbegriff unterschiedlich gefasst. Dabei handelt sich entweder um eine Kombination kosmischer und geschichtlicher Philosophie von Frieden und Krieg oder um einen dynamischen Transformationsprozess mit dem Ziel der Schaffung einer gerechten Ordnung.

\section{Friedenserfahrungen und -modelle in Asien}

Welche Friedenserfahrungen haben die einzelnen asiatischen Staaten in ihrer Staatsbildung gemacht und welche Modelle haben sich dort herausgebildet? Warum blieben große Staaten mit enormen sozialen Spannungen und/oder ethnischkulturellen Zerklüftungen wie beispielsweise Indien oder Indonesien, trotz der anhaltenden Konflikte in Teilgebieten (die Kaschmir-Frage in Indien sowie Aceh in Indonesien), von umfassenden Bürgerkriegen verschont? ${ }^{15}$

\subsection{Das konfuzianische Modell}

Die Lehre des Konfuzianismus strebt danach, eine ethische Sozialordnung zu bilden. Konfuzius suchte »politische Ordnung durch sittliche Ordnung « und »politische Harmonie durch moralische Harmonie beim einzelnen Menschen « zu erreichen. Der humanistische Gedanke, den Menschen am Menschen zu messen, verlangt, das wahre Selbst zu entdecken und mündet bei Konfuzius in die »Goldene Regel « der Gegenseitigkeit. ${ }^{16}$ Doch erst die Verbindung der Selbstverwirk-

14 Vgl. Harun Yahya, »Der Islam hat im Verlauf seiner ganzen Geschichte dem Mittleren Osten Frieden und Harmonie gebracht«, unter: <http://www.harunyahya.com/de/artikel15_islam.htm>.

15 Allerdings hat sich die politische Lage in beiden Konflikten seit dem Frühsommer 2005 stark entspannt. Indien betrachtet Kaschmir inzwischen als bilaterales Problem und nicht mehr nur als innere Angelegenheit. Indien und Pakistan sind sich bewusst, dass ein Frieden in Kaschmir entscheidend ist für den Wachstumskurs ihrer Volkswirtschaften und für eine effiziente Armutsbekämpfung ihrer Länder. Nach 29 Jahren Bürgerkrieg haben die indonesische Regierung und die Rebellen der Bewegung Freies Aceh (GAM) im August in Helsinki ein Friedensabkommen unterzeichnet. Das Friedensabkommen sieht eine Demilitarisierung der Rebellen und den Abzug indonesischer Truppen aus der Provinz vor. Siehe Christian Wagner, Jenseits von Kaschmir. Chancen einer Annährung zwischen Indien und Pakistan, SWP-Aktuell 46, Okt. 2004; Joachim Hoelzgen, »Der beklemmende Frieden «, Spiegel Online, 9.10.2005; "Friedensabkommen für Aceh unterzeichnet«, in: entwicklungspolitik online, 15.8.2005.

16 Diese Regel (Schu) besagt: »Was du nicht willst, das man dir tu, das füge keinem andern zu«. Siehe Robert E. Allison, »The Golden Rule as the Core Value in Confucianism and Christianity: Ethical Similarities and Differences «, in: Asian Philosophy, Vol. 2, Nr. 2, 1992, S.173-85. 
lichung mit der sittlichen Ordnung oder Zucht führt zur »zentralen Harmonie«. So ergäbe sich schließlich eine Gleichheit von Politik und Ethik. ${ }^{17}$ Dabei betonte Konfuzius vor allem die hohe Verantwortung des Herrschers. Mit dieser Idealvorstellung eines ausgewogenen Humanismus lassen sich die Bedeutung und Problematik eines konfuzianischen Modells in drei Aspekten erfassen.

Das Gesellschaftsbild von Konfuzius orientiert sich an einem hierarchisch gegliederten Gemeinwesen, in dem jeder gemäß seiner Stellung eine bestimmte Funktion mit entsprechenden Verhaltensnormen wahrnimmt. Wie Kinder gegenüber ihren Eltern, so sind Niedriggestellte gegenüber Höhergestellten zu Gehorsam verpflichtet. Kindlicher Gehorsam und Loyalität bilden daher die Grundtugenden. Die Merkmale dieser autoritären Kultur - Fleiß, Disziplin und Unterordnung - werden als asiatische Werte in die Wertedebatte eingebracht und propagiert. ${ }^{18}$ Allerdings steht das autoritäre Erbe der konfuzianischen Ordnungs- und Rechtstradition im Widerspruch zu internationalen Normen und Prinzipien wie Rechtsstaatlichkeit und Menschenrechte. Das traditionelle Gesetzesrecht Chinas stand im Dienste der Schaffung eines mächtigen Staates. Recht hatte in China kaum die Funktion des Schutzes individueller Freiheiten, weshalb ein traditionelles Misstrauen gegenüber institutionalisiertem formalem Recht existiert. Vor allem war der Gedanke einer Gleichheit vor dem Gesetz im Rahmen der konfuzianischen Soziallehre unvorstellbar, da Menschen als Träger ungleicher sozialer Rollen mit ungleichen Rechten und Pflichten verstanden wurden. ${ }^{19}$

Ein weiterer Aspekt des konfuzianischen Modells ist die Wertschätzung für die Bildung. Konfuzius war anerkannt als der erste große Erzieher, der seine Schüler zu unermüdlichem Lernen antrieb. ${ }^{20}$ Die auch von den Bauern bis auf den heutigen Tag geteilte Bewunderung für Bildung und Gelehrsamkeit, das traditionell respektierte Schul- und Bildungssystem, die Hoffnung, über Prüfungen politischen und wirtschaftlichen Erfolg zu erzielen, trugen und tragen dazu bei, das Prestige der Intellektuellen zu festigen, wie G. Paul schildert. ${ }^{21}$ F. Fukuyama hebt diesen Aspekt hervor, der in der Modernisierungstheorie als demokratiefördernd angesehen wird. ${ }^{22}$ Bildung ermöglicht den sozialen Aufstieg im hierarchischen politischen und gesellschaftlichen System und erhöht dadurch die Chancen der Beteiligung an politischen Entscheidungen. Die Betonung der Bildung hat jedoch die Wertvorstellungen und Einsichten über Sitten in konfuzianischer Tradition verengt. Nicht selten sorgten und sorgen die höhere Bewertung der »konfuzianischen« Gelehrten- und Beamtenschicht für Miss-

17 Ebd.

18 Der frühere Premierminister Singapurs, Lee Kuan Yew, und der früherer Premierminister Malaysias, Mahathir bin Mohamad, propagierten in den 1990er Jahren ihre autoritäre Politik als »den asiatischen Weg «. Siehe Lee Eun-Jeung, »Asien und seine sasiatischen Werte«", in: APuZ, Nr. 35-36, 25.8.2003, unter: <http://www.das-parlament.de/2003/35_36/Beilage/001.html>.

19 Sebastian Heilmann, »Volksrepublik China: Regierungssystem und politische Entwicklungen «, unter: <http://www.phil-fak.uni-duesseldorf.de/oasien/china/ info/bpb/china/body_i_198_5.html >.

20 »Lernen ist eine Tätigkeit, bei der man das Ziel nie erreicht und zugleich immer fürchten muss, das schon Erreichte wieder zu verlieren«. (Lunyu-Gespräche 8.17).

21 Gregor Paul, »Traditionelle Kultur, nationales Wirtschaftssystem und internationales Geschäft Anmerkungen zum deutsch-chinesischen Mitund Gegeneinander", in: China-Journal, Ausgabe 1, 2002-04, unter: <http://www.dcg.de/paul/kultwirt.html>

22 Francis Fukuyama, »Ich oder die Gemeinschaft«, in: Die Zeit, 11. 11. 1999. trauen und soziale Unruhe zwischen verschiedenen sozialen Schichten. ${ }^{23}$

Schließlich ist der Aspekt der Diesseitsorientierung in der konfuzianischen Kultur zu erwähnen. Konfuzianismus war weniger eine politische Lehre als eine moralische Lehre der Selbst-Kultivierung ${ }^{24}$ zum Streben nach dem Wohl der Familie und der Gesellschaft bis zum guten Regieren des Staates und zur Friedensstiftung unter dem Himmel. Das Zusammenleben in ethischer Verantwortung gegenüber der gruppendefinierten Gesellschaft spielte eine viel größere Rolle als Gottes- und Jenseitsglaube. ${ }^{25}$ Die konfuzianische Diesseitsorientierung erklärt viel. Einerseits, da es keine Aussicht auf ein Leben nach dem Tod gibt, lebt man nur einmal hier und jetzt. Dies ist ein starker Ansporn, das Beste aus dem eigenen Leben zu machen. Andererseits erschwert die Diesseitsorientierung die gemeinsame Ausrichtung auf ein alle verbindendes Ziel. Die verbreitete Rücksichtslosigkeit des digitalen Kapitalismus ${ }^{26}$ in China sowie die fehlende soziale Solidarität unter den Chinesen sind dadurch zu erklären.

Die konfuzianische Tradition hat mit ihren autoritären Elementen, massiven Investitionen in Bildung sowie Diesseitsorientierung eine wichtige Grundlage für die Wirtschaftsdynamik der Region geliefert. Dennoch fehlen gerade der konfuzianischen ethischen Ordnung konstruktive Auseinandersetzungen mit Konzepten wie Gleichheit und Rechtsstaatlichkeit, welche für die Bekämpfung struktureller Gewalt wie Ausbeutung, Ungerechtigkeit und eine wachsende Kluft zwischen Arm und Reich von zentraler Bedeutung sind.

\subsection{Das japanische Modell}

Mit der Öffnung gegenüber westlichen Einflüssen begann 1868 in Japan die Epoche der Modernisierung. Nach den Schrecken des Zweiten Weltkrieges sowie seiner Kriegsverbrechen ist Japan mit seiner Friedensverfassung durch wirtschaftlichen Wiederaufbau zu einem der reichsten demokratischen Industriestaaten geworden. ${ }^{27}$ Japan war und ist vor allem wirtschaftlich eine führende Macht in Asien. China, Taiwan, Südkorea und die südostasiatischen Länder (besonders Indonesien) sahen in Japan ein Modell für ihren wirtschaftlichen Modernisierungsprozess. Die dynamischen Entwicklungsprozesse in Süd- und Ostasien lassen sich mit dem Wildgänse-Modell erklären, indem Japan mit seiner offensiven Entwicklungshilfepolitik, Auslandsinvestitionen und kulturellen Diplomatie beim Auf-

23 Vgl. Paul, Traditionelle Kultur.

24 Der konfuzianische Idealmensch äußerte sich in der Erfüllung der überlieferten Pflichten. Siehe Max Weber, Die Wirtschaftethik der Weltreligionen. Band I, Konfuzianismus und Taoismus, VIII Resultat: Konfuzianismus und Protestantismus $(1915,1920)$, unter: <http://www.china1900.info/gedanken/ weberstudie/8resultate.htm>.

25 Wie Konfuzius damals zur Frage nach dem Tod sagte: »Wenn man über das Leben nichts weiß, wie kann man dann etwas über den Tod wissen?« (Lunyu 11.11)

26 Die Digitalität des angeblich futuristischen Kapitalismus erweist sich rasch als hinlänglich bekanntes Novum. Beschleunigung wird durch den Einsatz von elektronischer Datenverarbeitung und vom Datentransfer erzeugt. Vgl. Peter Glotz, Kulturkämpfe im digitalen Kapitalismus, München: Kindler, 1999.

27 Japan darf laut seiner Verfassung von 1947 keine Armee unterhalten, doch gelten die "Selbstverteidigungskräfte « (SDF) als modernste Streitkräfte Asiens. Vgl. Karl Grobe, »Japans Verfassung wird umgeschrieben«, in: Frankfurter Rundschau, 15.4. 2005. 
holprozess seiner unterentwickelten Nachbarn für die räumliche Expansion und die Vertiefung industrieller Zusammenarbeit in der Region eine Schlüsselrolle gespielt hat. ${ }^{28}$

Den Grundstein für das Aufblühen der Wirtschaft Japans legte die Meiji-Reform in der zweiten Hälfte des 19. Jahrhunderts. Kaiser Meiji erließ 1868 eine Verfassung, die sich an den modernen Grundsätzen der Gleichheit und Freiheit orientierte. Es folgte eine »Reform von oben « nach westlichem Vorbild, die sowohl die Bewahrung der Unabhängigkeit Japans als auch das Gleichziehen mit den anderen Großmächten zum Ziel hatte. ${ }^{29}$ Die Lernprozesse, welche die Japaner bei ihrer begeisterten Begegnung mit dem Westen (Kultur, Demokratie und Marktwirtschaft) durchliefen, ermöglichten den Aufstieg Japans als regionale Macht. Gleichwohl stieß seine Großmacht-Politik auf Grenzen und entfachte später mit seiner Politik des »Asien den Asiaten « den Pazifikkrieg gegen die USA und ihre Alliierten. Nach der Niederlage vermied es Japan, sich mit seiner kriegerischen Vergangenheit kritisch auseinander zu setzen. ${ }^{30}$ Während Japan den Krieg positiv zu werten sucht, stößt das in Gesellschaft und Politik mangelnde Verantwortungsgefühl immer wieder auf verbitterte Reaktionen in Ostasien, sowohl in der Öffentlichkeit als auch auf der Regierungsebene. ${ }^{31}$ Auf der Haben-Seite stehen eher wirtschaftliche Faktoren: Die Entwicklung Japans in der Nachkriegszeit sorgte sowohl für die Restrukturierung der japanischen Industrie als auch für die Industrialisierung seiner ostasiatischen Nachbarn und führte zu wirtschaftlicher Verflechtung.

\subsection{Das Indien-Modell}

Trotz seiner Größe, verbreiteter Armut, starker sozialer und ethnisch-kultureller Zerklüftung und gewalttätiger Konflikte ist Indien seit seiner Unabhängigkeit im Jahre 1947 nicht im Krieg auseinander gebrochen. Indien gilt als demokratisch konsolidierter Staat. Zeichen seiner Kontinuität als Demokratie sind das pluralistische Parteiensystem und die seit 1967 häufigen Regierungswechsel. ${ }^{32}$ Zusammen mit China und Brasilien gehört Indien zu den sich schnell entwickelnden Ländern, obwohl marktwirtschaftliche Reformen erst 1985 begannen.

28 Das von Akamatsu entwickelte »wild-geese-flying pattern of economic growth " geht von der These aus, dass die wirtschaftliche Kommunikation einer Nation mit Industrieländern die notwendige Voraussetzung ihrer Wirtschaftsentwicklung ist. Später bezieht Akamatsus Schüler Kojima die hinausgehenden Investitionen (FDI) fortgeschrittener Nationen ein und modifiziert das Wildgänsemodell als »catching up product cycle theory". Hwang Sun-Gil, Das Wildgänsemodell für Ost-Asien, Institut für sozialökonomische Handlungsforschung, Arbeitspapiere zur sozialökonomischen Ostasien-Forschung, Nr. 3. 1998.

29 Siehe Mark Hudson, Agriculture and language change in the Japanese Islands. In: Peter Bellwood/Colin Renfrew, Examining the farming/language dispersal hypothesis, Cambridge, 2002, pp. 311-317.

30 Martin Fackler, »Japan struggles with legacy. Questions of legacy lingers on «, in: International Herald Tribune (IHT), 15.8. 2005, p. 5.

31 In April 2005 gab es mehrere gewaltsame antijapanische Proteste in Südkorea und China. Auslöser der Proteste war ein kürzlich von Japan genehmigtes Schulbuch, in dem nach Ansicht Chinas und Koreas die Kriegsverbrechen Japans in China und Korea verharmlost werden. Die Demonstrationen führten zu einer neuen Belastung der Beziehungen zwischen China und Japan. »Tokio und Peking beharren auf gegenseitigen Entschuldigungen«, in: Spiegel Online, 18.4. 2005; Chikako Yamamotot, »Der unverstandene Nachbar«, in: TAZ, Nr. 7642, 18.4.2005, S 11 .

32 Die anhaltende Dominanz der die Unabhängigkeitsbewegung verkörpernden Kongresspartei machte bis 1967 Regierungswechsel auf nationaler bzw. unionsstaatlicher Ebene unmöglich. Siehe Bertelsmann Stiftung, »Den Wandel gestalten. Ländergutachten: Indien«, unter: $<$ http://www.bertelsmann-transformation-index.de/46.0.html>.
Als Stabilisierungsfaktor ersten Ranges im gelungenen wirtschaftlich-politischen Transformationsprozess Indiens ist die enorme gesellschaftliche Vielfalt zu erkennen. Laut J. Betz erschwerte die starke Fragmentierung die Herausbildung gesamtindischer Konfliktlinien. ${ }^{33}$ Indien gilt als historisches Modell für Multikulturalität. Das Land ist die volkreichste Demokratie mit über 700 Volksgruppen, ebenso vielen Dialekten und 30 Sprachen. Hier haben fast alle großen Religionsgemeinschaften über Jahrhunderte in engster Nachbarschaft gelebt: Hinduismus, Christentum, Islam und Buddhismus. M. Gandhis Strategie des »zivilen Ungehorsams « durch »gewaltfreien Widerstand « verlieh Indien das Image, ein Vorbild religiöser Toleranz zu sein. Im Vergleich zum Nachbar- und Bürgerkriegsstaat Sri Lanka fallen in Indien die geringer ausgeprägten Zerklüftungen und deren partielle Überlagerung auf. Inwiefern das multikulturelle Erbe des Landes für die Friedensstiftung von Bedeutung ist, hängt allerdings vom jeweiligen politischen Interesse ab, im gegebenen Fall davon, ob einem an der Stärkung kultureller Vielfalt oder vielmehr an der Herstellung von Homogenität gelegen ist. ${ }^{34}$

Auch die Kontinuität des demokratischen Systems erweist sich als sozio-politisch förderlicher Faktor für eine friedliche Konfliktbearbeitung. Von der Zerklüftung Indiens ging unter demokratischem Vorzeichen ein starker Zwang zu einer Politik der Mitte aus, für Säkularismus und sozialen Ausgleich. Mit seinem Föderalismus stellt Indien daher einen der wenigen leidlich funktionierenden Bundesstaaten der Welt dar, dem es gelang, regionalen Interessen und Loyalitäten legale Ausdrucksmöglichkeiten zu verschaffen, separatistische Eliten einzubinden und periphere Konflikte zu mildern. ${ }^{35}$ Die demokratische Kontinuität hat vor allem die Wende zur Marktwirtschaft begünstigt, die politisch geschickt, ohne übermäßige Eile und unter Schonung politisch wichtiger gesellschaftlicher Gruppen sowie der Armen, umgesetzt wurde mit dem Ergebnis, dass keine ernst zu nehmende Gruppierung heute wirtschaftspolitisch den Status quo ante anstrebt.

Des Weiteren sind die Charakteristika der staatlichen Herrschaft sowie die daraus resultierende Ambivalenz zwischen Modernität und Tradition zu erwähnen. Indiens Herrschaftssystem ist eine Mischung aus rationalen und charismatischen Elementen, wobei insgesamt der rationale Typus überwiegen dürfte. ${ }^{36}$ Wenngleich die traditionalen Elemente (z.B. Herrschaft einer Kaste) eher auf der kommunalen Ebene vorkommen, scheint die Moderne in Indien gerade durch das gemischte Herrschaftssystem ein janusköpfiges Gesicht zu zeigen. Die Moderne wird entweder als politisches Instrument genutzt (mit der Idee, dass der homogene Nationalstaat das notwendige Ambiente für Demokratie, Frieden und Wohlstand anbietet) oder als ein Projekt angestrebt. Pointiert gesagt konkurrieren heute in Indien zwei dominante Zweckdefinitionen: Indien als »Einheit in Vielheit « (Nehru) und Indien als »homogener Einheitsstaat« (Sarvarkar). Welches Ziel sich durchsetzen wird,

33 "Friedenserfahrungen in Asien, Afrika und Lateinamerika«, unter: <http://sicherheitspolitik.bundeswehr.de/2/14/4.php>

34 Michael Gottlob, »Leitkultur am Ganges? Geschichtspolitik und Revisionismus in Indien «, in: Internationale Schulbuchforschung, Nr. 23, 2001, S. $465-476$.

35 Friedenserfahrungen in Asien, Afrika und Lateinamerika, a.a.O.

36 Max Weber, Wirtschaft und Gesellschaft, Teil I, Kap. III, Die Typen der Herrschaft, 1922. 
hängt davon ab, inwieweit es den jeweiligen Vertretern der beiden »Projekte « gelingt, ihre Mittel möglichst effektiv dafür einzusetzen (Mobilisierung von Unterstützung, Zuteilung von Wohlfahrtschancen, außenpolitischer Erfolg etc). ${ }^{37}$

Die multikulturelle Tradition und die gesellschaftliche Fragmentierung, das solide demokratische System sowie das gemischte Herrschaftssystem stellen wichtige Faktoren für die gelungene Transformation Indiens dar. Allerdings kann das indische Friedensmodell durch innenpolitische Faktoren (den stärker werdenden religiös-politischen Fundamentalismus und die relativ ineffiziente Armutsbekämpfung) gefährdet werden. ${ }^{38}$

\subsection{Das Indonesien-Modell}

Indonesien verdient nicht nur wegen seiner Größe und geostrategischen Bedeutung Aufmerksamkeit, sondern auch wegen der spezifischen Ausprägung, die der Islam hier genommen hat. Er weist vielfältigere Erscheinungsformen und größere Toleranz auf als dies in einigen arabischen Staaten der Fall ist. ${ }^{39}$ Vor allem seit den Wahlen vom Juni 1999 befindet sich Indonesien auf dem Weg der demokratischen Transformation. Die Wahlen 2004 erwiesen sich als Durchbruch zur Demokratie. ${ }^{40}$ Der in den späten 1960er Jahren begonnene wirtschaftliche Transformationsprozess ging dem politischen voraus und verhalf Indonesien zum Aufstieg von einem der ärmsten Länder der Welt zu einem »lower-middle income country«.

Eines der wichtigsten Charakteristika und zugleich eine Herausforderung für Indonesien ist das Zusammenleben in multikulturellen Gemeinschaften. Für B. Tibi verkörpert Indonesien ein Modell, in dem verschiedene religiöse und kulturelle Gemeinschaften einander respektieren und friedlich miteinander leben. ${ }^{41}$ Im scharfen Kontrast zur Türkei gewährleistet die auf der Pancasila gründende Verfassung Kultusfreiheit für die anerkannten Religionen (Islam, Christentum, Buddhismus, Hinduismus). Obwohl der Islam während des Freiheitskampfs gegen die holländische Kolonialherrschaft identitätsstiftend wirkte, spielte er in der Verfassung nach der Unabhängigkeit 1945 eine vergleichsweise unbedeutende Rolle. ${ }^{42}$ Auch haben die Erfahrungen mit den freien Wahlen seit 1999 gezeigt, dass der politische Islam bislang keine breite Basis hat.

Des Weiteren setzt Indonesien mit seinem Demokratisierungsprozess ein unverwechselbares Signal, dass der Islam demokratiefähig ist. ${ }^{43}$ Nach Ansicht N. Othmans sind die Indonesier den Malaysiern in Bezug auf das islamische Denken

37 Andreas Metzger, »Ist Indien 'modern ? Max Weber und die indische Politik «, unter: <http://parapluie.de/archiv/indien/weber/> (24.08.05).

$38 »$ Frieden ist nicht alles, aber ohne Frieden ist alles nichts«, St. Johanne Friedenstage 2002 am Beispiel Indien, SoliTat, INTERSOL, Dez. 2002; Klaus Schäfer, »Religionsfreiheit in Indien unter Druck «, Evangelischer Missionswerk in Deutschland, 30.8.2005

39 »Aktuelle Situation in der islamischen Welt«, Bundeszentrale für politische Bildung, unter: <http://www.bpb.de/publikationen/NVSS5G,0,0,Aktuelle_ Situation_der_islamischen_Welt.html $>$.

40 Bertelsmann Stiftung, »Den Wandel gestalten. Ländergutachten: Indonesien«; Moritz Kleine-Brockhoff, Indonesien: Durchbruch für die Demokratie, Friedrich Ebert Stiftung-Analyse, Dez. 2004.

41 Bassam Tibi, »Indonesia, a Model for the Islamic Civilization in Transition to the 2lst Century«, in: FAZ, 27.10. 1995.

42 Vgl. Infodienst Indonesien und Osttimor Nr. 29, Mitte Juli - Ende September, 2004.

43 Arnfrid Schenk, »Geht doch. Indonesien hat einen neuen Präsident gewählt«, in: Die Zeit, 21.9.2004. und die intellektuelle Kultur um Lichtjahre voraus. Den Indonesiern ist gelungen, durch kritische und holistische Ansätze dem Islam einen Modernisierungsimpuls zu geben. Als Beispiel nennt Othman die Erfahrungen in den islamischen Dorf- und Gemeindeschulen. Dort sieht man in den islamischen Rechtsgelehrten mehr als religiös versierte Gelehrte, nämlich eine kulturelle und soziale Kraft. ${ }^{44}$ Merkmale des indonesischen Islam, der sich zwischen Modernisten und Traditionalisten bewegt und günstig für die demokratische Entwicklung ist, sind Toleranz, soziale Mobilität und Achtung vor dem Gesetz. Gerade im Verbund mit traditioneller südostasiatischer Harmonie- und Konsenskultur haben diese Merkmale dazu beigetragen, den Spielraum für Islamisten erheblich einzuschränken, so G. Schubert. Gleichzeitig haben sie einen Beitrag zum Überleben der demokratischen Institutionen und Verfahren in Indonesien geleistet, trotz vieler Rückschläge. ${ }^{45}$

Allerdings bleibt unklar, inwiefern eine Kombination wirtschaftlicher Konsolidierung und praktizierter Toleranz als Friedensmodell nicht nur für die islamische Zivilisation, sondern auch für die Verständigung zwischen Kulturen dienen kann. Angesichts der Dominanz konservativer Interessengruppen und ethnischer, religiöser und regionaler Konfliktlinien befindet sich die indonesische Demokratie immer noch in einer prekären Situation. S. Anwar spricht von einer schleichenden Islamisierung, in der einige Regionen mittels ihres Autonomie-Rechts das Scharia-Gesetz forciert haben (z. B. West-Java und Madura). Religion wird leicht als politisches Instrument populistischer Bewegungen missbraucht. ${ }^{46}$

\subsection{Das wirtschaftliche Integrationsmodell}

Handelsdaten zeigen, dass die Jahre 1970-2000 durch den weltwirtschaftlichen Aufstieg Ostasiens und zunehmende innerasiatische Handelsintegration geprägt waren. ${ }^{47}$ Ein neues Zentrum der Weltwirtschaft bildet sich heraus: China. Die wachsende wirtschaftliche Bedeutung der ASEAN-Staaten hat mehr mit der Dynamik Ostasiens als mit dem ASEAN-Regelwerk zu tun. ${ }^{48}$ Vor allem Indien hat 2004 einen Meilenstein erreicht: Sein Handelsvolumen mit den Ländern der asiatischpazifischen Region überholte erstmalig dasjenige mit seinem US- und seinen europäischen Handelspartnern zusammen. ${ }^{49}$ Asien ist eine Region mit großer ökonomischer Dynamik und Kooperation geworden, d.h. wesentliche Bedingungen für Integration sind vorhanden. Neben der Frage nach der Form (Freihandelsabkommen oder verstärkte Institutionalisierung mit einheitlicher Währung) stellt sich die nach der Machbarkeit.

44 Martina Sabra, »Der Prophet war für die Gleichberechtigung «, Interview mit Norani Othman, in: $E+Z, 07 / 2005$.

45 Gunter Schubert, Wie demokratiefähig ist der Islam? Konrad Adenauer Stiftung e.V. Archive, unter: <http://www.kas.de/db_files/dokumente/ auslandsinformationen/7_dokument_dok_pdf_250_1.pdf $>$.

46 Michael Vatikiotis, »Islamizing Indonesia«, 3-4. 9. 2005, in: IHT, S. 5; Sebastian Braun/Felix Heiduk/Kay Möller, »Indonesien: Demokratie, Regierbarkeit und nationaler Zusammenhalt«, SWP-Studie/S 06, Feb. 2005.

47 Patrick Ziltener, Ostasiatische oder pazifische Handelsdynamik? Eine Analyse von UNCTAD-Handelsdaten, 1970-2000, MPIfG Working Paper 02/9, Juli 2002.

48 Ebd.

49 Anand Giridharadas, »Rising India remains torn between East and West «, in: $I H T, 15.8 .2005$, S. 7. 
Zwei Variationen stehen zur Debatte. Die Idee Greater China als Wirtschaftsraum für Entwicklung und Wachstum steht für eine Struktur mittels verstärkter Handels- und Kapitalbeziehungen zwischen ethnischen Chinesen im geografischen Raum von Festlandschina, Hongkong, Macao, Taiwan bis zu den südostasiatischen Staaten. Das Konzept ist, so seine Verfechter, international wettbewerbsfähig, lässt die intraregionalen Entwicklungspotentiale zum Tragen kommen und wird den Veränderungen der politischen Rahmenbedingungen gerecht. Diese Idee stößt auf normative und praktische Probleme. ${ }^{50}$ Hier hat der Begriff oft den Beigeschmack einer »imperialen «, maßgeblich mit China assoziierten Pax Sinica, die den nichtchinesischen Anrainerstaaten (Südkorea und Japan), aufgezwungen wird. Auch sind politische Faktoren entscheidend für seine gesamte Entwicklung. Dazu gehören die Ostasienpolitik der USA, das Verhältnis zwischen den USA und China, die japanisch-chinesischen Beziehungen, die Entwicklung in Russland, das Verhältnis zwischen Indien und China sowie zwischen Indien und Pakistan, die Entwicklung auf der koreanischen Halbinsel und die Taiwan-Frage. Zudem ist durch die verstärkte wirtschaftliche Integration sowie die daraus folgende gegenseitige Abhängigkeit ein Steuerungsproblem für die jeweiligen Regierungen entstanden. ${ }^{51}$

Als zweites Konzept stellt sich seit Anfang der 1990er Jahre die Idee einer asiatischen Freihandelszone. Sie fördert ihren Anhängern zufolge das wirtschaftliche Wachstum, stärkt die Wirtschaftsposition Asiens (gegenüber EU und NAFTA) und erhält Frieden, Stabilität und Wohlstand. Die Herausbildung regionaler Kooperationsmechanismen in Gestalt der East Asia Economic Group (EAEG) und später des East Asian Economic Caucus (EAEC) bis zur ASEAN-Plus-Three (China, Japan und Südkorea) hat gezeigt, dass die asiatischen Akteure ihre Gemeinsamkeiten im Hinblick auf geteilte Interessen und Konflikte bewusst wahrnahmen und anzupacken versuchten. ${ }^{52}$ Allerdings kommt die Regionalisierung Ostasiens politisch und institutionell nur schleppend in Gang. Die Gründe sind ökonomischer und politischer Natur. Strukturelle Disparitäten in der Region, Unterschiede der politischen Systeme, krasse Ungleichheiten in der Einkommensverteilung sowie in der sektoralen Entwicklung der Volkswirtschaften haben Liberalisierungs-Vereinbarungen deutlich erschwert. Politisch pikant war nicht nur die vorgeschlagene Führungsrolle Japans im EAEC, die besonders Südkorea nicht goutieren konnte. Aus Sicht der meisten ostasiatischen Staaten gilt vor allem unter ökonomischen Gesichtspunkten der Ausschluss der USA und Australiens als unzumutbar. Hinzu kommt Dissens über die Rolle der ASEAN-Plus-Three und eines Führungslandes. Malaysia und China neigen dazu, einen ostasiatischen Block gegen die USA und die NAFTA zu instrumentalisieren. Japan hinge-

50 Über die Idee Greater China siehe Elena Meyer-Clement/Gunter Schubert, Greater China - Idee, Konzept und Forschungsprogramm, Greater China Occasional Papers No 1, Okt. 2004, Universität Tübingen; Carsten HermannPillath, »Festlandfieber «: Politisch-ökonomische Aspekte der Beziehungen Taiwans zum chinesischen Festland, Gerhard-Mercator-Universität GH Duisburg.

51 Vgl. Hermann-Pillath, Festlandfieber, ebd.

52 Die Gemeinsame Erklärung zum ASEAN-Plus-Three-Gipfel in Manila 1999 hält fest, dass das Gipfelforum nach Möglichkeiten der Kooperation sucht. Vgl. Markus Hund / Nuria Okfen, Vom East Asian Economic Caucus (EAEC) to ASEAN-Plus-Three, unter: <http://www.politik.uni-trier.de/forschung/ coll_1.pdf >; M. Mayer, Wechselkurspolitik und Wachstumsstabilität in SüdOstasien, Trends East Asia, Studie Nr. 4 (Juni 2004). gen reagiert sehr sensibel auf Widerstand seitens der USA und kann oft seiner angestrebten Rolle in Ostasien nicht gerecht werden. ${ }^{53}$ Obgleich China regionale Vormacht werden will, ist es momentan nicht in der Lage, sein eigenes Innovations-, Produktions- und Dienstleistungssystem zu entwickeln und in Asien und der Welt zu verbreiten. Immerhin bietet das technologisch starke Japan seinen Nachbarn Stimuli an und gilt deshalb als ökonomisch wichtiger als China.

\section{Frieden schaffen in Asien-Aufarbeitung der Vergangenheit als Priorität}

Die Analyse der wichtigsten Modelle zeigt, dass Asien zwar positiv zum Frieden beigetragen hat, der Region jedoch eine umfassende Definition der Bedeutung Asiens in der Welt fehlt. Die Beziehungen der asiatischen Länder zueinander sind hauptsächlich von der Wirtschaft und dem weltpolitischen Geschehen diktiert worden.

Die Würdigung der Modelle richtet den Blick auf ihre politische Tauglichkeit, die Probleme des jeweiligen Modells und nicht zuletzt auf ihren Umgang mit kollektiver Erinnerung. Denn diese Modelle mit ihrer starken Betonung der Harmonie stoßen sich an einer mangelhaften Praxis, die auch jüngst wieder für teils gewalttätige Konflikte sorgte (antijapanische Übergriffe in China und Südkorea). Weit weniger als etwa Westeuropa hat Asiens Bevölkerung Erfahrungen mit Gewalt aufklärend und konstruktiv verarbeitet, weder im Inneren (etwa die Verantwortung der eigenen Regierung für die Millionen Opfer des »Großen Sprungs « oder der »Kulturrevolution « in China und des Massenmordes an bis zu einer Million mutmaßlicher Kommunisten unter dem jungen Suharto-Regime) noch in den Außenbeziehungen (Japans Umgang mit seiner Aggression während des Zweiten Weltkrieges bzw. NankingMassaker und Zwangsprostitution). Peking blockiert die Erinnerung im Inneren und damit auch die offen-konstruktive Aufarbeitung der Beziehung zu Japan.

Gründe für das Fehlen einer kritischen, systematischen und versöhnlichen Aufarbeitung der eigenen und gemeinsamen Vergangenheit liegen nicht zuletzt in der Großmachtpolitik und den Überresten der Mentalität des Kalten Krieges. Trotz der Blockfreienbewegung hat sich das heterogene Asien grundsätzlich am dominierenden geopolitischen Diskurs orientiert und entsprechend reagiert. Ihm fehlt bisher eine autonome regionale Sicht und Programmatik, um politische Solidarität, wirtschaftliche Zusammenarbeit und sozial-kulturelle Beziehungen in der Region zu entwickeln. Das wirft einen Schatten auf die Chancen Asiens, zum Weltfrieden beizutragen. So ist bei der Schilderung der Modelle und ihrer wünschenswerten Weiterentwicklung besonders darauf zu achten, welchen Platz sie der Erinnerung zuweisen.

53 Ebd. 